\section{Präanalytische Phase}

W. G. Guder

München, Deutschland

Synonym(e) Prämetrologische Phase

Englischer Begriff preanalytical phase; premetrological phase; pre-examination procedures (phase)

Definition Alle die $>$ Probe betreffenden Vorgänge und Arbeitsschritte einer laboratoriumsmedizinischen Untersuchung von der Patientenvorbereitung bis zur Entnahme der analytischen Portion im Analysesystem.

Beschreibung In den letzten Jahren ist das Bewusstsein für die Bedeutung der $>$ Probennahme, des Transports und der > Probenvorbehandlung für die Qualität des laboratoriumsmedizinischen Befundes stark gewachsen. Nach einer jüngeren Übersicht haben 40-75\% aller Fehler von Laboratoriumsbefunden in dieser Phase ihre Ursache. Dies ist wesentlich durch die immer besser werdende Standardisierung und Qualitätssicherungsmaßnahmen in der analytischen Phase bedingt. Dies führte zur Definition der präanalytischen Phase, die auch im Zeitablauf des gesamten diagnostischen Prozesses mehr als $50 \%$ ausmacht. Sie umfasst folgende Prozesse:
- Wahl der Untersuchung

- Auswahl der Probe, des Zeitpunktes und des anatomischen Orts der Probennahme

- Patientenvorbereitung (z. B. Diät, Körperlage, Aufklärung)

- Gewinnung des Untersuchungsmaterials inklusive aller dazu notwendigen Materialien und Prozesse

- Transport und Aufbewahrung der Probe

- Probenvorbereitung mit Gewinnung der analytischen Probe

Die einzelnen Prozeduren und deren Dokumentation sind mittlerweile fester Bestandteil der BÄK-Richtlinien ( $\vee$ Bundesärztekammer) sowie der DIN-EN-ISO-Norm 15185.

\section{Literatur}

Bonini P, Plebani M, Ceriotti F, Rubolli F (2002) Errors in laboratory medicine. Clin Chem 48:691-698

Bundesärztekammer (2008) Richtlinie der Bundesärztekammer zur Qualitätssicherung laboratoriumsmedizinischer Untersuchungen. D Ärztebl 105:C301-C315. www.bundesaerztekammer.de

DIN EN ISO 15189 (2007) Medizinische Laboratorien - Besondere Anforderungen an die Qualität und Kompetenz. Beuth-Verlag, Berlin

Guder WG, Narayanan S (2015) Pre-examination procedures in laboratory diagnostics. Walter de Gruyter, Berlin/Boston

Guder WG, Narayanan S, Wisser H, Zawta B (2009) Diagnostic samples: from the patient to the laboratory, 4. Aufl. Wiley-Blackwell, Weinheim 